\title{
Dauricine inhibits viability and induces cell cycle arrest and apoptosis via inhibiting the PI3K/Akt signaling pathway in renal cell carcinoma cells
}

\author{
SHUWEI ZHANG ${ }^{1,2}$, YU REN ${ }^{2}$ and JIANXIN QIU ${ }^{1}$ \\ ${ }^{1}$ Department of Urology, Shanghai General Hospital of Nanjing Medical University, Shanghai 200080; \\ ${ }^{2}$ Department of Urological Surgery, Ningbo Urology and Nephrology Hospital, Ningbo, Zhejiang 315000, P.R. China
}

Received April 18, 2017; Accepted October 31, 2017

DOI: $10.3892 / \mathrm{mmr} .2018 .8732$

\begin{abstract}
Renal cell carcinoma (RCC), which is derived from the proximal tubules of nephrons, is one of the most common solid cancers. Due to its inherent insensitivity to radiotherapy and chemotherapy, surgery remains the only curative strategy for RCC. Therefore, a novel strategy for treating RCC is urgently needed. This study aims to investigate the effects of dauricine, a bisbenzylisoquinoline alkaloid, in RCC cells and the underlying mechanisms of its action. The effects of dauricine on viability, cell cycle distribution and apoptosis in RCC cells were determined in vitro by MTT assay, flow cytometry and nucleosome ELISA assay, respectively. Mechanism studies were performed by analyzing related proteins using western blotting assays. We show that dauricine effectively inhibits the viability of four RCC cell lines (786-O, Caki-1, A-498 and $\mathrm{ACHN}$ ). In addition, dauricine induces cell cycle arrest at the G0/G1 phase in RCC cells. Dauricine also induces apoptosis via the intrinsic pathway, since caspase- 9 and caspase- 3 but not caspase- 8 activation was detected after the treatment. Moreover, dauricine was able to inhibit the PI3K/Akt signaling pathway. Our findings suggest inhibitory effects of dauricine in renal cancer cells and provide a better understanding of its underlying mechanism. Our findings suggest that dauricine could be a potential therapeutic agent for treating RCC.
\end{abstract}

\section{Introduction}

Renal cell carcinoma (RCC), one of the most common solid cancers found in adult kidneys, is an epithelial carcinoma derived from the proximal tubules of nephrons (1). RCC accounts for $\sim 90 \%$ of kidney carcinomas and $3 \%$ of all adult

Correspondence to: Dr Jianxin Qiu, Department of Urology, Shanghai General Hospital of Nanjing Medical University, 100 Hai Ning Road, Shanghai 200080, P.R. China

E-mail: jasonqiu@sjtu.edu.cn

Key words: renal cell carcinoma, dauricine, apoptosis, cell cycle, $\mathrm{PI} 3 \mathrm{~K} / \mathrm{Akt}$ malignancies (1). Due to its inherent insensitivity to radiotherapy and chemotherapy, surgery remains the only curative strategy for RCC (2). However, approximately one-third of patients develop metastases after surgery (3). Therefore, novel therapeutic strategies are urgently needed.

In recent years, considerable attention has been paid to natural products for preventing or treating cancers due to their safety. Over the past 30 years, over $70 \%$ of all drugs approved by the Food and Drug Administration (FDA) for cancer have originated from natural products or traditional medicine (4). Dauricine, a bisbenzylisoquinoline (BBIQ) alkaloid isolated from the rhizome of Menispermum dauricum DC, has been found to yield various pharmacological results, such as anti-arrhythmic and anti-inflammatory effects (5). Moreover, several studies have indicated that dauricine has potent antitumor activities, including inducing apoptosis, repressing viability and overcoming drug resistance in tumor cells (6). However, the effect of dauricine on $\mathrm{RCC}$ remains to be elucidated.

In the present study, we investigated the biological effects and mechanisms underlying dauricine action in RCC cells. We found that dauricine inhibits viability in cells and induces cell cycle arrest at the G0/G1 phase and apoptosis via the intrinsic pathway in RCC cells. The mechanisms of dauricine's action included the repression of anti-apoptotic $\mathrm{Bcl}-2$ proteins and the PI3K/Akt signaling pathway.

\section{Materials and methods}

Cell culture and reagents. The human RCC cell lines 786-O, Caki-1, A-498 and ACHN were purchased from the American Type Culture Collection (ATCC; Manassas, VA, USA). The cells were cultured in RPMI-1640 medium containing 10\% fetal bovine serum (both from Gibco; Thermo Fisher Scientific, Inc., Waltham, MA, USA) and 1\% (v/v) penicillin-streptomycin (Sigma-Aldrich; Merck KGaA, Darmstadt, Germany) at $37^{\circ} \mathrm{C}$ in a humidified atmosphere containing $5 \% \mathrm{CO}_{2}$. Dauricine was purchased from Solarbio Biotechnology Co., Ltd. (Beijing, China). 3-(4,5-dimethyl-2-thiazolyl)-2,5-diphe nyl-2H-tetrazolium bromide (MTT) and propidium iodide (PI) were purchased from Sigma-Aldrich Chemicals (St. Louis, MO, USA). Primary antibodies against caspase-8, caspase-3, 
caspase-9, cleaved PARP, Bcl-2, p21, and Bax were purchased from Cell Signaling Technology, Inc. (Danvers, MA, USA). Primary antibodies against cyclin D1, cyclin-dependent kinase 2 (CDK2), CDK4, p-Akt, Akt, p-PI3K, and PI3K were all purchased from Abcam (Cambridge MA, USA), and primary antibodies against GAPDH and donkey anti-rabbit and sheep anti-mouse immunoglobulin were purchased from Sigma-Aldrich. All other chemicals not specifically mentioned here were purchased from Sigma-Aldrich; Merck KGaA.

Cell viability assay. Cell viability was evaluated by MTT assay. Briefly, cells were seeded in a 96-well plate $\left(5 \times 10^{3}\right.$ cells/well) and then treated with various doses of dauricine for $24 \mathrm{~h}$. A total of $50 \mu \mathrm{l}$ of MTT solution $(5 \mathrm{mg} / \mathrm{ml})$ was added, and the cells were incubated for another $4 \mathrm{~h}$. The medium was then removed, and $200 \mu \mathrm{l}$ of DMSO was added to each well. The absorbance of the solutions was measured on a BioTek microplate reader at $595 \mathrm{~nm}$. The relative cell viability was normalized to the control, which was treated with $0.1 \%$ DMSO.

Cell cycle analysis. Cells were harvested after treatment with dauricine and fixed in ethanol. The cells were washed with PBS and stained with propidium iodide (BD Biosciences, Franklin Lakes, NJ, USA) for 30 min in PBS supplemented with RNase at room temperature in the dark. The cell cycle distribution was then evaluated using FACSVerseTM (Beckman Coulter Fullerton, CA, USA), and the data were analyzed using FlowJo V10 (Tree Star, Inc., Ashland, OR, USA).

Nucleosome ELISA assay for the detection of apoptosis. For apoptosis assays, cells were seeded at a density of $1 \times 10^{4}$ cells/well into 96 -well plates at $37^{\circ} \mathrm{C}$ overnight and treated with various doses of dauricine for $24 \mathrm{~h}$. Cells were subsequently harvested and treated with the Nucleosome ELISA kit (Sigma-Aldrich; Merck Millipore, Darmstadt, Germany) according to the manufacturer's instructions.

Caspase activity assay. The activity of caspases was measured using a caspase activation kit (R\&D Systems, Minneapolis, MN, USA) according to the manufacturer's instructions. Briefly, cell lysates were prepared after treatment with various doses of dauricine and incubated with the supplied reaction buffer and the colorimetric substrates at $37^{\circ} \mathrm{C}$ for $2 \mathrm{~h}$ in the dark. Then, the absorbance of the solutions was measured on a BioTek microplate reader (BioTek Instruments, Winooski, VT, USA) at $405 \mathrm{~nm}$.

Western blot analyses. Total protein was lysed in RIPA lysis buffer (Invitrogen Life Technologies, Carlsbad, CA, USA) and then quantified with a BCA kit. Equal amounts of proteins were resolved on $12 \%$ SDS-PAGE gels and transferred onto PVDF membranes. Membranes were incubated with primary antibodies overnight at $4{ }^{\circ} \mathrm{C}$ followed by HRP-conjugated secondary antibodies at room temperature for $1 \mathrm{~h}$; signals were visualized by ECL reagent (Pierce, Rockford, IL, USA).

Statistical analyses. All the experiments were carried out at least three times. The data were analyzed using GraphPad Prism 6.0 software. The results are shown
Table I. $\mathrm{IC}_{50}$ values of dauricine in renal carcinoma cells.

\begin{tabular}{lr}
\hline Cell lines & $\mathrm{IC}_{50}$ \\
\hline $786-0$ & $13.42 \pm 2.1 \mu \mathrm{M}$ \\
Caki-1 & $8.46 \pm 1.3 \mu \mathrm{M}$ \\
A-498 & $18.28 \pm 0.6 \mu \mathrm{M}$ \\
ACHN & $11.36 \pm 1.5 \mu \mathrm{M}$ \\
\hline
\end{tabular}

Data represent mean \pm standard deviation $(n=3)$. $\mathrm{IC}_{50}$, half maximal inhibitory concentration.

as the mean \pm standard deviation (SD), and the differences were measured using one-way analysis of variance (ANOVA) followed by Tukey's test. $\mathrm{P}<0.05$ was considered to indicate a statistically significant difference.

\section{Results}

Dauricine inhibited the viability of renal carcinoma cells. To investigate the cytotoxicity of dauricine, four different renal carcinoma cell lines (786-O, Caki-1, A-498, and ACHN) were treated with various concentrations of dauricine $(0,5$, $10,15,20 \mu \mathrm{M}$ ) for $24 \mathrm{~h}$, followed by MTT assay. As shown in Fig. 1, dauricine significantly decreased cell viability in a dose-dependent manner, and the $\mathrm{IC}_{50}$ values of dauricine in different cell lines are listed in Table I. Caki-1 and A-498 cells were the most sensitive and the least sensitive to dauricine, respectively. These two cell lines and 786-O cells were used for the subsequent studies.

Dauricine induced cell cycle arrest at the G0/G1 phase. MTT assay revealed that dauricine inhibits the viability of RCC cells. To better understand the underlying mechanism, the effects of dauricine on cell cycle were analyzed using flow cytometry. Caki-1, A-498 and 786-O cells were treated with different doses of dauricine (10 and $20 \mu \mathrm{M})$ for $24 \mathrm{~h}$. As shown in Fig. 2A, incubation with dauricine triggered cell cycle arrest at the G0/G1 phase as well as a reduction in the $\mathrm{S}$ phase in a dose-dependent manner. Moreover, cell cycle-related proteins, such as cyclin D1, CDK2, and CDK4, were decreased, and p21 was increased after treatment with dauricine (Fig. 2B).

Dauricine induced apoptosis via the intrinsic pathway in renal carcinoma cells. To measure apoptosis induced by dauricine, nucleosome ELISA assays were used. As shown in Fig. 3A, dauricine treatment led to an increase in apoptosis in a dose-dependent manner. The activation of caspases plays a very important role in the process of apoptosis. There are two pathways that lead to apoptosis, namely, the extrinsic pathway and the intrinsic pathway. The extrinsic and intrinsic pathways are initiated by caspase- 8 and caspase- 9 , respectively. Activation of both caspase- 8 and caspase- 9 can lead to the activation of caspase-3, which finally leads to apoptosis. Therefore, we investigated whether dauricine activated any of these caspases using a colorimetric caspase activity assay and western blot analyses. As shown in Fig. 3B and C, 

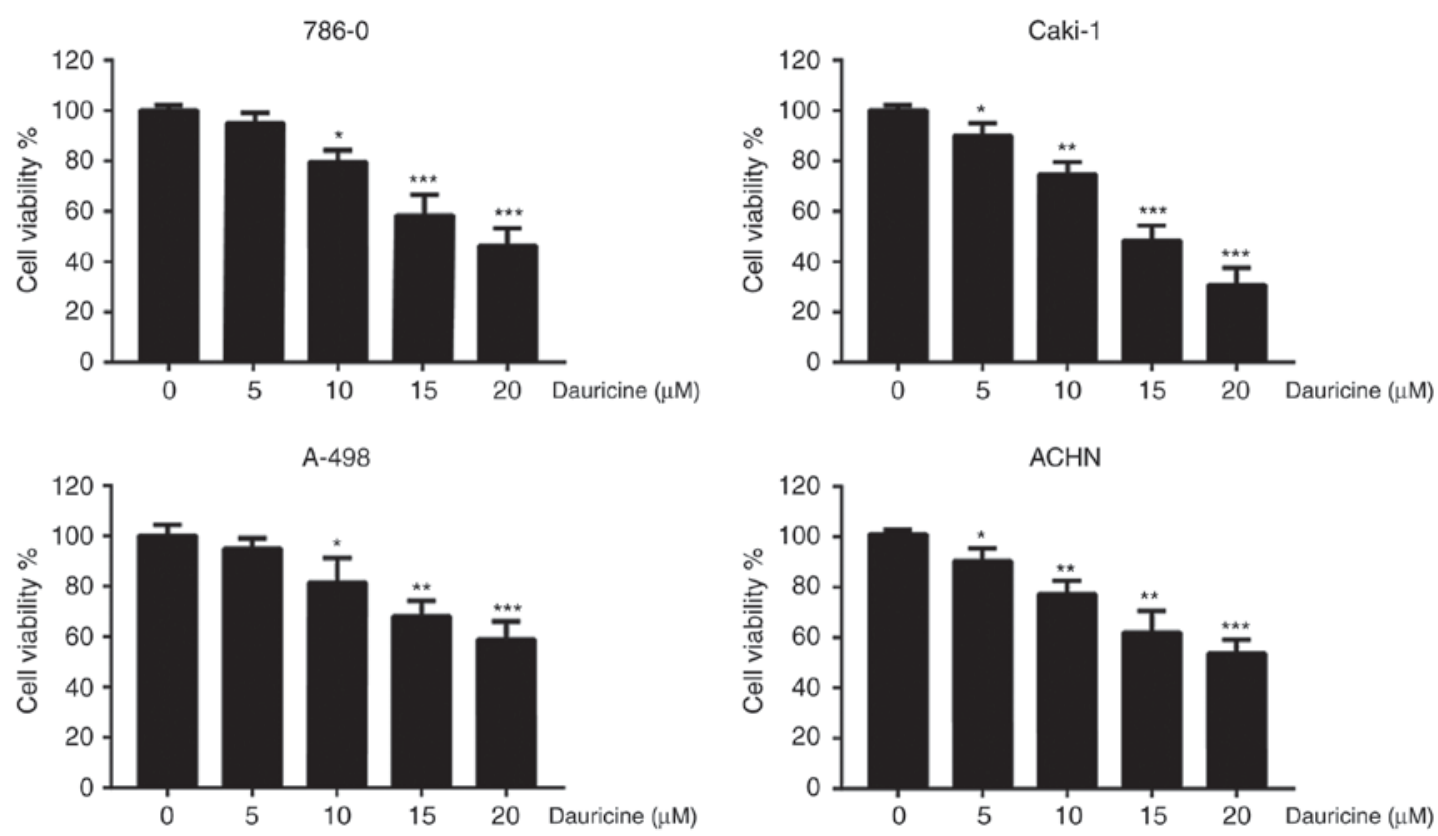

Figure 1. Effects of dauricine treatment on renal carcinoma cell viability. Various renal carcinoma cell lines (786-O, Caki-1, A-498, and ACHN) were treated with various doses of dauricine for $24 \mathrm{~h}$, and MTT assays were performed to determine cell viability. The data are expressed as the mean \pm SD of three independent experiments. Significance was determined by Student's t-test $\left({ }^{*} \mathrm{P}<0.05,{ }^{* *} \mathrm{P}<0.01\right.$ and ${ }^{* * * *} \mathrm{P}<0.001$ vs. untreated control).
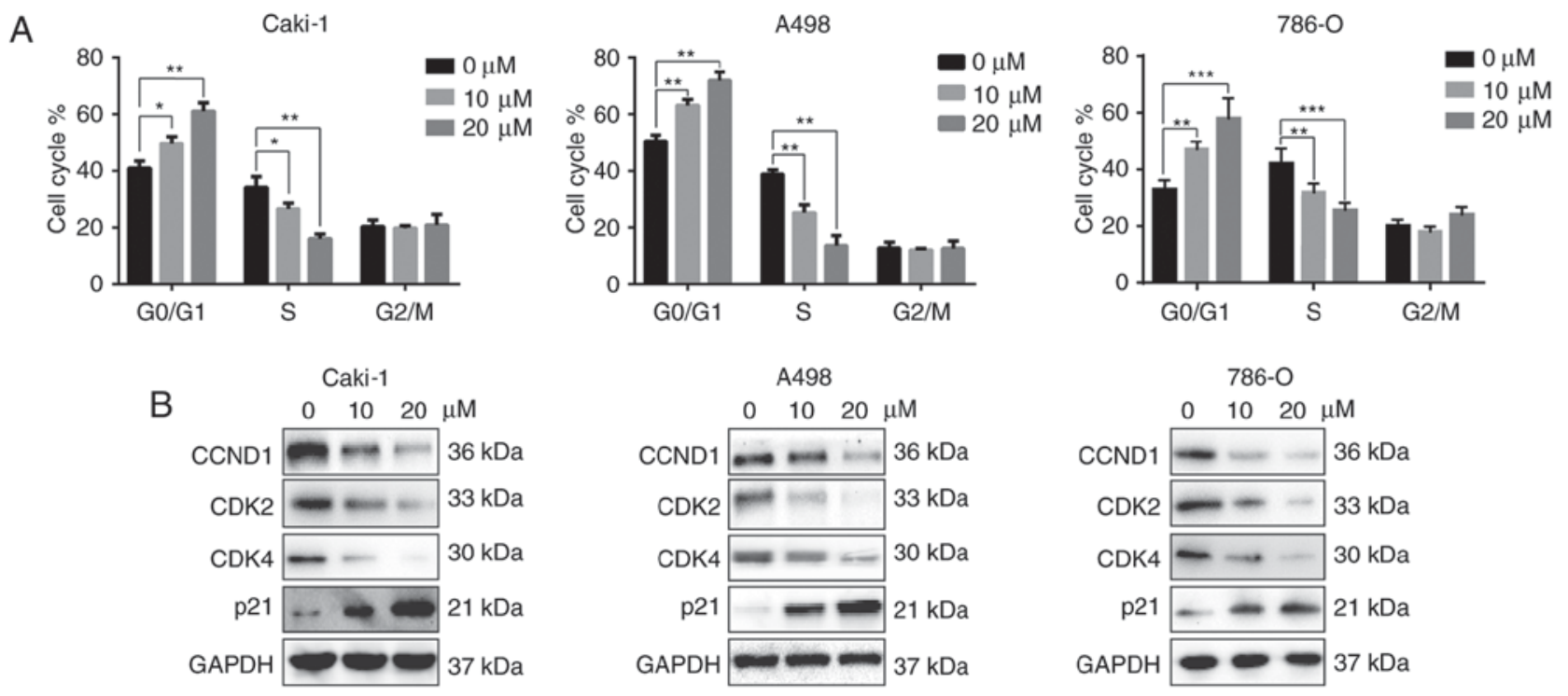

Figure 2. Dauricine induces cell cycle arrest at the G0/G1 phase. (A) Caki-1, A-498 and 786-O cells were treated with various doses of dauricine for 24 h; cells were then stained with PI and subjected to flow cytometry analysis. (B) Caki-1 and A-498 cells were treated with various doses of dauricine for 24 h, and cellular lysates were subjected to western blot assays with the indicated antibodies. The data are expressed as the mean \pm SD of three independent experiments. Significance was determined by Student's t-test $\left({ }^{*} \mathrm{P}<0.05,{ }^{* *} \mathrm{P}<0.01\right.$ vs. untreated control).

activation of caspase- 3 and caspase- 9 but not caspase- 8 was observed after incubation with dauricine. Besides the cleavage of caspase-3, we also observed the expression of pro-caspase- 9 was decreased which indicated the activation of caspase-9 (7,8) (Fig. 3C). In addition, cleavage of PARP, which is a substrate of caspase-3, was observed (Fig. 3C). The Bcl-2 family proteins are well-known regulators of the intrinsic apoptotic pathway. We also found that dauricine was able to downregulate the anti-apoptotic Bcl-2 and Mcl-1 proteins in a dose-dependent manner (Fig. 3C). Moreover, the pro-apoptotic protein Bax was increased by dauricine in a dose-dependent manner (Fig. 3C). These data suggest that dauricine induced apoptosis mainly through the intrinsic apoptotic pathway in renal carcinoma cells.

Dauricine suppresses the PI3K/Akt signaling pathway in renal carcinoma cells. The PI3K/Akt signaling pathway plays a critical role in cell survival and protecting cancer cells from apoptosis (9). Therefore, we investigated whether dauricine could affect the PI3K/Akt signaling pathway. Caki-1, A-498 and 786-O cells were treated with various concentrations of dauricine for $24 \mathrm{~h}$; then, cellular lysates were subjected to 
A

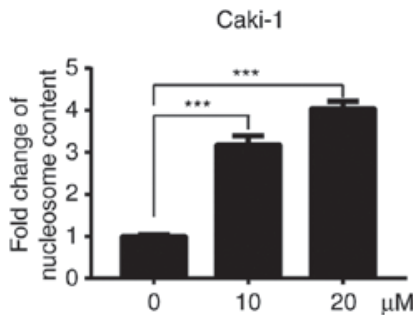

B
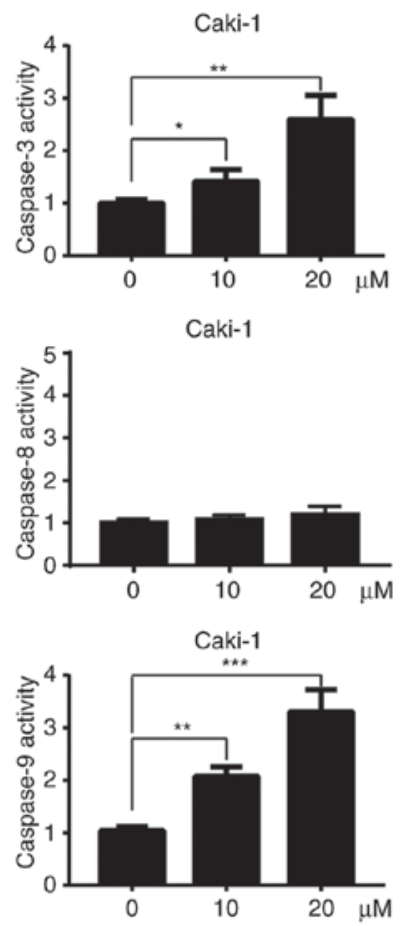
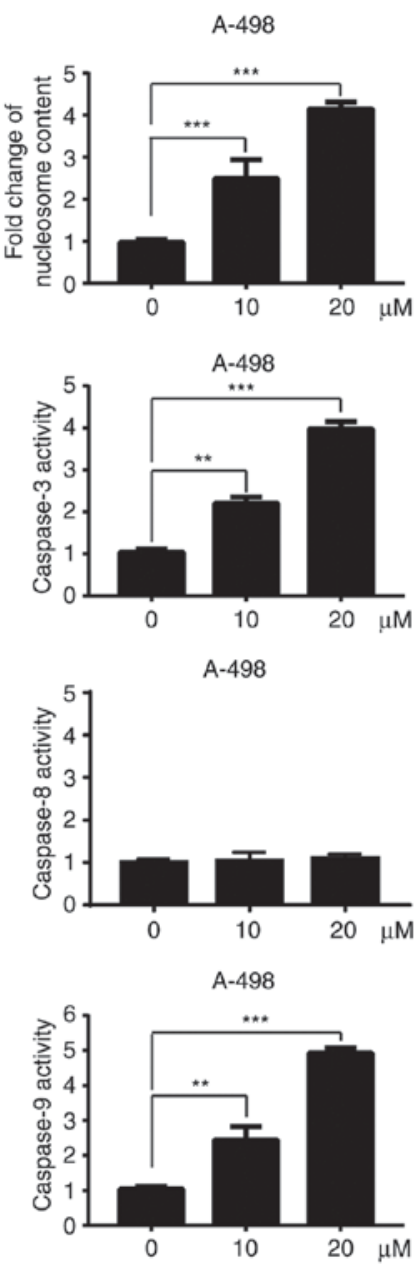

A-498
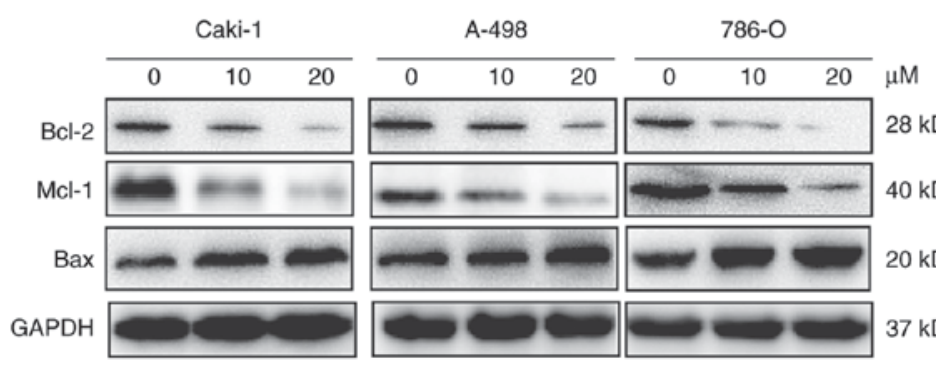

$28 \mathrm{kDa}$

$40 \mathrm{kDa}$

$20 \mathrm{kDa}$

$37 \mathrm{kDa}$

Figure 3. Dauricine induces apoptosis via the intrinsic pathway. Caki-1, A-498 and 786-O cells were treated with various doses of dauricine for 24 h. (A) Cell apoptosis was measured using a nucleosome ELISA kit. (B) Cellular lysates were assayed for in vitro caspase-3, -8 and -9 activities using DEVD-pNA, IETD-pNA and LEHD-pNA as substrates, respectively, at $37^{\circ} \mathrm{C}$ for $1 \mathrm{~h}$. The released fluorescent products were quantified. (C) Cellular lysates were subjected to western blot analyses with the indicated antibodies. The data are expressed as the mean \pm SD of three independent experiments. Significance was determined by Student's t-test $\left({ }^{*} \mathrm{P}<0.05,{ }^{* *} \mathrm{P}<0.01,{ }^{* * *} \mathrm{P}<0.001\right.$ vs. untreated control).

western blot analysis. As shown in Fig. 4A, the activation of PI3K and Akt was suppressed by dauricine. To further investigate the role of dauricine in PI3K inhibition, we examined its effect with or without the endotoxin LPS, which is a potent activator of the PI3K/Akt signaling pathway. The cells were incubated with $2 \mu \mathrm{g} / \mathrm{ml}$ LPS or $20 \mu \mathrm{M}$ dauricine for $24 \mathrm{~h}$. As depicted in Fig. 4B, stimulation with LPS significantly induced Akt activation, and dauricine could repress the activation of 
A

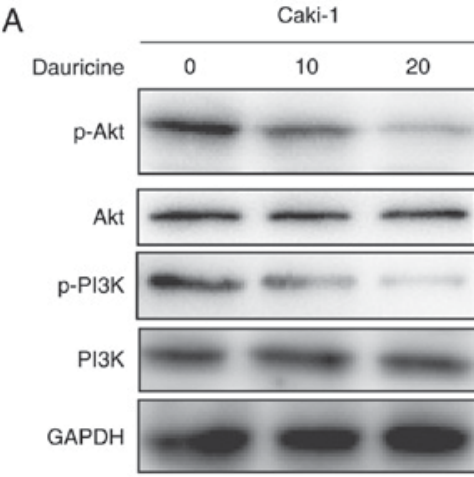

B

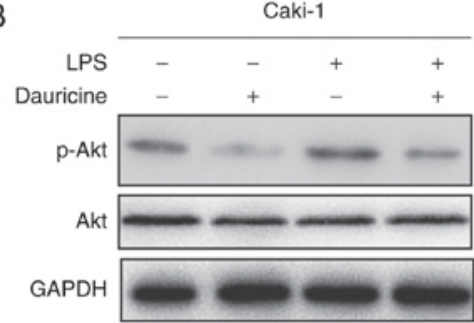

A-498

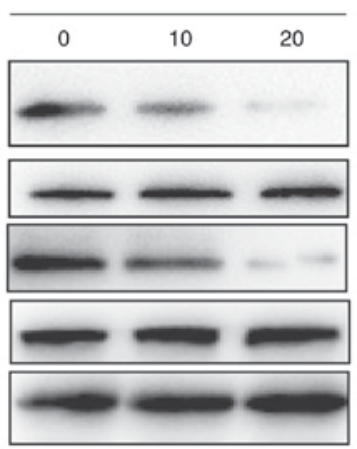

A-498

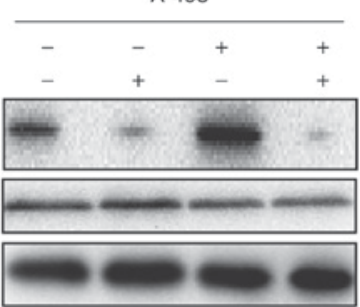

$768-0$

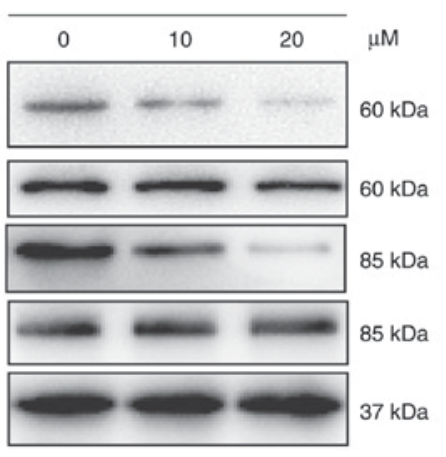

$768-0$

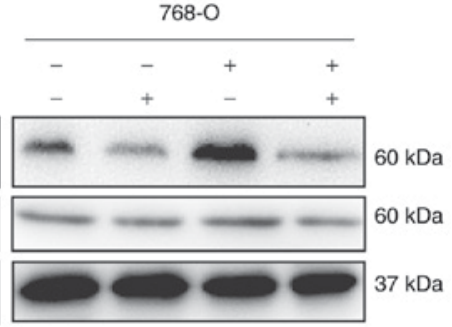

Figure 4. Dauricine represses the PI3K/Akt signaling pathway. (A) Caki-1, A-498 and 786-O cells were treated with various doses of dauricine for $24 \mathrm{~h}$. Cellular lysates were subjected to western blot assays with the indicated antibodies. (B) Caki-1, A-498 and 786-O cells were treated with dauricine (20 $\mu \mathrm{M})$ or LPS $(2 \mu \mathrm{g} / \mathrm{ml})$ for $24 \mathrm{~h}$, and cellular lysates were subjected to western blot analyses with the indicated antibodies.

Akt. These data suggest that dauricine may exert antitumor effects through the inhibition of PI3K/Akt.

\section{Discussion}

Currently, the treatment of RCC includes chemotherapy, radiotherapy, immunotherapy, targeted therapy and surgery. Unfortunately, RCC is insensitive to radiotherapy and chemotherapy. Surgery is mainly used during the early metastasis of RCC, thereby limiting its application. Immunotherapy and targeted therapy have not yielded reliable remission in RCC patients and need to be further developed $(10,11)$. Therefore, novel therapeutic agents for treating RCC are urgently needed.

BBIQs are a large and diverse family of natural alkaloids that can be isolated from many plants. These alkaloids have received considerable attention due to their diverse pharmacologic activities, such as anti-inflammatory, cardiovascular, and antitumor effects (12-14). Dauricine is a BBIQ alkaloid isolated from the root of Menispermum dauricum DC (15). Several studies have found that dauricine possesses potent antitumor activities. For example, dauricine could inhibit angiogenesis in breast cancer cells (16). Dauricine could also overcome doxorubicin resistance in human leukemia cells (12). However, the effects of dauricine in RCC cells have not yet been studied.

In the present study, we investigated the effects of dauricine on RCC cells and the molecular events underlying the effects. Our results showed that treatment with dauricine significantly reduced the viability of four RCC cell lines (786-0, Caki-1, A-498, and ACHN) in a dose-dependent manner. Moreover, dauricine was found to cause cell cycle arrest at the G0/G1 phase. The cell cycle is a series of events leading to cell division and replication and is strictly regulated by cyclins and CDKs. It has been documented that cyclin D1 and CDKs are critical for cells to cross the G1/S threshold (17). Our data demonstrated that cyclin D1, CDK2 and CDK4 were downregulated and that $\mathrm{p} 21$ was upregulated after treatment with dauricine. These findings were similar to a previous study in which dauricine induced cell cycle arrest at the G0/G1 phase in colon cancer cells (18).

Apoptosis, also known as programmed cell death, is considered as one of the most potent natural defenses against cancer, and the ability to escape from apoptosis is a hallmark of cancer (19). Apoptosis is initiated by caspases, a family of cysteine aspartyl-specific proteases. There are two pathways that lead to apoptosis, namely, the extrinsic and intrinsic pathways, which are initiated by caspase- 8 and caspase- 9 , respectively (20). According to our findings, the activation of caspase- 3 and caspase- 9 was detected, but that of caspase- 8 was not affected in the cells treated with dauricine. Dauricine treatment also led to increased levels of cleaved PARP. Furthermore, dauricine was found to decrease the expression of Bcl-2 and Mcl-1 dose-dependently. These data suggest that dauricine induces apoptosis via the intrinsic pathway in RCC cells.

Deregulation of the PI3K/Akt signaling pathway is implicated in the initiation and development of various human malignancies, such as NSCLC, bladder cancer, RCC, breast cancer and colon cancer $(21,22)$. Many studies have indicated that the pro-apoptotic effects of some antitumor agents are tightly associated with the repression of the PI3K/Akt signaling pathway $(9,21)$. In addition, aberrant activation of the PI3K/Akt pathway is also related to insensitivity to chemotherapeutic agents (23). These findings suggest that targeting the PI3K/Akt signaling pathway may serve as a promising strategy for the treatment of cancers. In the present study, we investigated the possible role of the PI3K/Akt pathway in dauricine-induced apoptosis in RCC cells and found that phosphorylated PI3K and Akt were markedly decreased after dauricine treatment, 
but the total levels of PI3K and Akt were not changed. In addition, dauricine also inhibited the activation of PI3K/Akt induced by LPS. These findings suggest that dauricine exerts its antitumor effect via repressing the PI3K/Akt signaling pathway in RCC cells.

In conclusion, we investigated the antitumor potential of dauricine in RCC cells. We found that dauricine reduced viability in RCC cells, induced cell cycle arrest at the G0/G1 phase and induced apoptosis via the intrinsic pathway. Dauricine-induced cell cycle arrest was associated with the downregulation of cyclin D1, CDK2, and CDK4 and the upregulation of $\mathrm{p} 21$. In addition, dauricine-induced apoptosis was associated with the activation of caspase- 9 and caspase-3, and the expression of anti-apoptotic Bcl-2 proteins was downregulated. Furthermore, dauricine also inhibited the activation of the PI3K/Akt signaling pathway. These findings suggest that dauricine may be a promising chemotherapeutic agent for treating RCC. Nevertheless, further study is needed to completely elucidate the mechanisms of the antitumor effects of dauricine.

\section{References}

1. Ridge CA, Pua BB and Madoff DC: Epidemiology and staging of renal cell carcinoma. Semin Intervent Radiol 31: 3-8, 2014.

2. Chow WH, Devesa SS, Warren JL and Fraumeni JF Jr: Rising incidence of renal cell cancer in the United States. JAMA 281: 1628-1631, 1999.

3. Escudier B, Porta C, Schmidinger M, Rioux-Leclercq N, Bex A, Khoo V, Gruenvald V and Horwich A; ESMO Guidelines Committee: Renal cell carcinoma: ESMO clinical practice guidelines for diagnosis, treatment and follow-up. Ann Oncol 27 (Suppl 5): v58-v68, 2016.

4. Collins I and Workman P: New approaches to molecular cancer therapeutics. Nat Chem Biol 2: 689-700, 2006.

5. Yang XY, Jiang SQ, Zhang L, Liu QN and Gong PL: Inhibitory effect of dauricine on inflammatory process following focal cerebral ischemia/reperfusion in rats. Am J Chin Med 35: 477-486, 2007.

6. Law BY, Chan WK, Xu SW, Wang JR, Bai LP, Liu L and Wong VK: Natural small-molecule enhancers of autophagy induce autophagic cell death in apoptosis-defective cells. Sci Rep 4: 5510, 2014.

7. Li P, Nijhawan D, Budihardjo I, Srinivasula SM, Ahmad M, Alnemri ES and Wang X: Cytochrome $c$ and dATP-dependent formation of Apaf-1/caspase-9 complex initiates an apoptotic protease cascade. Cell 91: 479-489, 1997.
8. Hu Y, Benedict MA, Ding L and Núñez G: Role of cytochrome $c$ and dATP/ATP hydrolysis in Apaf-1-mediated caspase-9 activation and apoptosis. EMBO J 18: 3586-3595, 1999.

9. Yu R, Yu BX, Chen JF, Lv XY, Yan ZJ, Cheng Y and Ma Q: Anti-tumor effects of atractylenolide I on bladder cancer cells. J Exp Clin Cancer Res 35: 40, 2016.

10. Kanesvaran R and Tan MH: Targeted therapy for renal cell carcinoma: The next lap. J Carcinog 13: 3, 2014.

11. Derosa L, Albiges L, Massard C, Loriot Y, Fizazi K and Escudier B: Safety of available treatment options for renal cell carcinoma. Expert Opin Drug Saf 15: 1097-1106, 2016.

12. He QY, Meng FH and Zhang HQ: Reduction of doxorubicin resistance by tetrandrine and dauricine in harringtonine-resistant human leukemia (HL60) cells. Zhongguo Yao Li Xue Bao 17: 179-181, 1996 (In Chinese).

13. Marshall SJ, Russell PF, Wright CW, Anderson MM, Phillipson JD, Kirby GC, Warhurst DC and Schiff PL Jr: In vitro antiplasmodial, antiamoebic, and cytotoxic activities of a series of bisbenzylisoquinoline alkaloids. Antimicrob Agents Chemother 38: 96-103, 1994.

14. Qian JQ: Cardiovascular pharmacological effects of bisbenzylisoquinoline alkaloid derivatives. Acta Pharmacol Sin 23: 1086-1092, 2002.

15. Li YH and Gong PL: Neuroprotective effects of dauricine against apoptosis induced by transient focal cerebral ischaemia in rats via a mitochondrial pathway. Clin Exp Pharmacol Physiol 34: 177-184, 2007.

16. Tang XD, Zhou X and Zhou KY: Dauricine inhibits insulin-like growth factor-I-induced hypoxia inducible factor 1alpha protein accumulation and vascular endothelial growth factor expression in human breast cancer cells. Acta Pharmacol Sin 30: 605-616, 2009.

17. Salazar-Roa M and Malumbres M: Fueling the cell division cycle. Trends Cell Biol 27: 69-81, 2017.

18. Yang Z, Li C, Wang X, Zhai C, Yi Z, Wang L, Liu B, Du B, Wu H, Guo X, et al: Dauricine induces apoptosis, inhibits proliferation and invasion through inhibiting NF-kappaB signaling pathway in colon cancer cells. J Cell Physiol 225: 266-275, 2010.

19. Hanahan D and Weinberg RA: Hallmarks of cancer: The next generation. Cell 144: 646-674, 2011.

20. Brenner C, Galluzzi L, Kepp O and Kroemer G: Decoding cell death signals in liver inflammation. J Hepatol 59: 583-594, 2013.

21. Zhang L, Wu J, Ling MT, Zhao L and Zhao KN: The role of the $\mathrm{PI} 3 \mathrm{~K} / \mathrm{Akt} / \mathrm{mTOR}$ signalling pathway in human cancers induced by infection with human papillomaviruses. Mol Cancer 14: 87, 2015.

22. Porta C and Figlin RA: Phosphatidylinositol-3-kinase/Akt signaling pathway and kidney cancer, and the therapeutic potential of phosphatidylinositol-3-kinase/Akt inhibitors. J Urol 182: 2569-2577, 2009.

23. Faes S and Dormond O: PI3K and AKT: Unfaithful partners in cancer. Int J Mol Sci 16: 21138-21152, 2015. 